\title{
Effects of overnight radiation with monochromatic far-red and blue light on flower budding and expression of flowering-related and light quality-responsive genes in Eustoma grandiflorum
}

\author{
Tomoki Shibuya, Takuma TaKahashi, Shun Hashimoto, Manabu NishiYama and Yoshinori Kanayama ${ }^{\dagger}$ \\ ( Graduate School of Agricultural Science, Tohoku University, Sendai 980-8572, Japan )
}

\begin{abstract}
The effects of overnight radiation with monochromatic light on flower budding using far-red- and blue light-emitting diodes (FR and B, respectively) were investigated in Eustoma grandiflorum under controlled temperature and 8-h sunlight. Flower budding was promoted by FR and B, while it was not observed under short-day conditions with an 8-h photoperiod during the treatment period. The flower budding with FR was earlier than with B. Expression analysis of flowering-related genes and potential light quality-responsive genes was performed to find genes involved in light quality-dependent regulation of flowering. The levels of EgFTL and EgSOC1L mRNA increased preceding flower budding with FR and B, suggesting that both genes could be involved in the effects on flowering brought about by far-red and blue lights. Later and lower expression of these genes induced by B compared to FR could be associated with the weak promotion of flowering by blue light in E. grandiflorum. In potential light quality-responsive genes, the level of $E g B T B-P O Z 1$ mRNA peaked with $E g F T L$ with FR. Collectively, $E g F T L$ and $E g S O C 1$ likely promote flowering with FR and B, and $E g B T B-P O Z 1$ might be possibly involved in the promotion of flowering with FR.
\end{abstract}

Key words: Flower budding, FLOWERING LOCUS T, Light-emitting diodes, SUPPRESSOR OF OVEREXPRESSION OF CONSTANS1

\section{Introduction}

Flowering is known to be affected by photoperiod and light quality. Long-day conditions are required for flowering in long-day cut flowers, and although flowering is generally inhibited under the short-day conditions of fall and winter, the production of cut flowers is possible at such times through the use of artificial light. Blue, red, and far-red wavelengths are crucial in plant photomorphogenesis and flowering (Shibuya and Kanayama, 2014). In Arabidopsis, a long-day model plant, flowering is promoted by blue and far-red light but not by red light (Eskins, 1992), and the molecular mechanism pertaining to flowering-related genes has been reported. CONSTANS (CO) directly activates the transcription of FLOWERING LOCUS $T$ (FT), which is a major floral integrator in Arabidopsis. $C O$ expression, which is regulated by the circadian clock-regulated proteins, exhibits higher expression in the afternoon, while $\mathrm{CO}$ protein is stabilized by blue light and far-red light via some photoreceptors in the afternoon under long-day conditions to induce $F T$ expression (Srikanth and Schmid, 2011). The complex of CONSTITUTIVE PHOTOMORPHOGENESIS 1 (COP1) and SUPRESSOR OF PHYA-105 (SPAs) actively degrades CO at night and phyA interacts with SPA1 and represses COP1-SPA1 complex activity under far-red light (Sheerin et al., 2015). It is a high-irradiance response to a far-red light signal via phyA

Received; October 18, 2018

Accepted; May 29, 2019

†Corresponding Author: yoshinori.kanayama.a7@tohoku.ac.jp

DOI: 10.2480/agrmet.D-18-00041 on photoperiodic flowering (Casal et al., 1997). Besides, blue light receptor cryptochromes (CRY1 and CRY2) interact with SPA1 and repress COP1-SPA1 function on light depending manner (Li et al., 2011; Liu et al., 2011; Zuo et al., 2011). SUPPRESSOR OF OVEREXPRESSION OF CONSTANS1 (SOC1) is also a major floral integrator in Arabidopsis and it integrates photoperiod and gibberellic acid (GA) signals to promote flowering via SQUAMOSA PROMOTER BINDING PROTEIN-LIKE (Jung et al., 2012). Based on such molecular mechanisms, flowering is promoted by the relatively high expression of $F T$ and $S O C 1$ in the afternoon (e.g., Zeitgeber time (ZT) 8) by far-red and blue light in Arabidopsis under conditions comparatively similar to the long-day treatment in cut flower production, including weak light irradiation at night (Hori et $a l ., 2011)$. On the other hand, it is suggested that the influence of light quality on flowering is diverse in horticultural plants (Hamamoto et al., 2003).

In previous studies, long-day treatment has been performed using incandescent lamps to control flowering for cut flower production; however, the use of incandescent lamps, which have low energy efficiency and short life, is not environmentally friendly (Khan and Abas, 2011). Thus, light emitting diodes (LEDs) are attracting attention as an alternative light source because of their high energy efficiency and long life. In addition, LEDs have features of compactness and low operating temperature as an advantage in supplemental lighting for various purposes (Hikosaka et al., 2013; Murakami et al., 2013). On the other hand, unlike incandescent lamps, LEDs emit monochromatic light although white light can be irradiated by mixing colors or using a phosphor. 
The flowering of Eustoma grandiflorum (Raf.) Shinn., used in this study, is reportedly promoted by a low red:far-red ratio obtained using fluorescent lamps (Sato et al., 2009; Yamada et al., 2008; 2009). However, few studies have compared the effects of far-red and blue LED monochromatic light on flowering. Furthermore, there is a lack of information regarding the effects on flowering of far-red and blue light at the molecular level, an important factor in understanding the regulatory mechanisms involved in response to light quality.

Several studies that have investigated flowering response in cut flowers have not assessed the effect of monochromatic light; they used polychromatic light in long-day treatments (e.g., Sato et al., 2009; Yamada et al., 2008; 2009). In previous experiments, far-red light promoted flowering in Gypsophila paniculata L. and, interestingly, far-red light, when supplemented with weak red light (which normally inhibits flowering), enhanced flowering to an even greater degree, suggesting the existence of complex mechanisms of flowering response to light quality (Nishidate et al., 2012). In addition to such a complex phenomenon, there would be different mechanisms of promotion and suppression of flowering under each light color. Moreover, it is necessary to determine the most effective monochromatic light and to compare its effects with those of mixed light. Therefore, the effects of monochromatic light quality on flowering and expression of flowering-related genes should first be investigated to gain a comprehensive understanding of the floral response to light quality. In the present study, we investigated the effects of far-red and blue LED (FR and $\mathrm{B}$, respectively) radiation under controlled growth conditions during the dark period on the flower budding of E. grandiflorum, an important long-day cut flower. We compared their effect on the expression of $E$. grandiflorum homologs of the floral integrators FT and SOC1 (Nakano et al., 2011), and on genes potentially responsive to light quality to explore light quality-dependent regulation of flowering.

\section{Materials and methods}

\subsection{Plant materials}

E. grandiflorum 'Piccolosa Snow' (Sakata Seed, Yokohama, Japan), grown in a phytotron (sunlight type) set at $22{ }^{\circ} \mathrm{C} / 15^{\circ} \mathrm{C}$ under sunlight, was used in this study. Seeds were sown in peat plates on March 24, 2014 and grown in cell trays filled with Sumi-Soil (Sumika Agrotech, Osaka, Japan) for approximately 20 days after germination. Individual seedlings with between one and two nodes were planted in pots filled with the mixture of culture soil, compost, and vermiculite. All plants were grown under short-day conditions with an 8-h photoperiod (sunlight from 09:00 to 17:00, darkness from 17:00 to 09:00 under shading) for 2 months to promote vegetative growth before beginning the LED radiation treatment. The treatment began on June 30, 2014 using seedlings with two and three nodes, and lasted 14 weeks. The plants were grown under sunlight from 09:00 to 17:00 and under either FR, B, or no LED light (SD) with shading between 17:00 and 09:00. The illumination units using shell-type LEDs (trial product, Shibasaki Inc., Chichibu, Japan) were suspended at a height of $1.1 \mathrm{~m}$. The spectra of the LEDs are shown in Hori et al. (2011); FR and B had peaks at 740 and $465 \mathrm{~nm}$, respectively. The photon flux densities (average $\pm \mathrm{SE}$ of nine measurement points) were $10.0 \pm$ 0.08 and $8.8 \pm 0.13 \mu \mathrm{mol} \mathrm{m} \mathrm{m}^{-2} \mathrm{~s}^{-1}$ at the soil surface for FR and B, respectively (OPTOMETER P-9710, Gigahertz-Optik, Puchheim, Germany). The actual temperature (average \pm SD of each 30 minutes from June 30 to July 29) were $21.9 \pm 1.29$ and $17.1 \pm 0.78{ }^{\circ} \mathrm{C}$ for day and night, respectively (TR-51S, T\&D Corporation, Matsumoto, Japan). A slow-release fertilizer (CDU TamagokaseiS555; Co-op Chemical, Tokyo, Japan) was added to the culture every 2 weeks, and pesticides were used as required. The daily cumulative values of PPFD, relative humidity, and $\mathrm{CO}_{2}$ concentration were not measured during the cultivation period.

\subsection{Gene expression analysis}

Mature leaves were sampled at 17:00 every 2 weeks, selecting evenly from the upper to the lower leaves, and the samples were stored at $-80{ }^{\circ} \mathrm{C}$. RNA was extracted using a Cica Geneus RNA Prep Kit for Plant (Kanto Chemical, Tokyo, Japan). Removal of genomic DNA from the RNA samples and reverse transcription were performed using a QantiTect Reverse Transcription Kit (Qiagen, Hilden, Germany). Real-time PCR was performed using a CFX Connect Real-Time System (Bio-Rad, Hercules, USA) with a Thunderbird SYBR qPCR Kit (Toyobo, Osaka, Japan). The following thermocycler program was run: 40 cycles at $95^{\circ} \mathrm{C}$ for $15 \mathrm{~s}, 55.6{ }^{\circ} \mathrm{C}(E g F T L$, EgSOC1L), $56.8^{\circ} \mathrm{C} \quad(E g F L C L)$, or $60.0^{\circ} \mathrm{C} \quad(E g G A 20 o x$, EgGA2ox, EgBTB-POZ1) for $30 \mathrm{~s}$, and $72{ }^{\circ} \mathrm{C}$ for $30 \mathrm{~s}$. Melting curves were used to confirm single products. Threshold cycles were determined using CFX Manager Software (Bio-Rad), and the constitutively expressed actin gene was used as a reference for normalizing gene expression. The following primer sets were designed using Primer3Plus (http://primer3plus.com): 5'-AAGCCCTAGTGACCCAAATCTC-3' and 5'-CTCGTACCACACGATTTCTTGG-3' for EgFTL (accession number AB565512); 5'-GATCAAGGCTCCATCAACACAG-3' and 5'-TCCAAGTAACTTTGCATCTCCTCTC-3' for EgSOC1L (AB565509); and 5'-CAGGAGCTTGAAACTGCAAAGAG-3' and 5'-GCTGGAATAGGACTTCTGGACAAC-3' for actin $(E g A C T, \mathrm{AB} 565514)$. The nucleotide sequences of the primer sets for $E g F L C L, E g G A 20 o x, E g G A 20 x$, and $E g B T B-P O Z 1$ were the same as those used by Nakano et al. (2011) and Takemura et al. (2015).

\section{Results and discussion}

\subsection{Promotion of flowering by far-red and blue light}

Since continuous lighting in the dark period was the most effective treatment condition for far-red rich bulb-type fluorescent lamps on flowering in a study on E. grandiflorum (Sato et al., 2009), and there are other horticultural studies employing similar treatment conditions (e.g., Miyamae et al., 2015; Nishidate et al., 2012), continuous lighting was employed at first to reveal the influence of light quality in the present study. In the future, it would be necessary to consider effective methods even under shorter irradiation conditions. The timing of flowering was determined by recording the number of days elapsed until flower budding, and the number of nodes formed by the same time. The number of days and nodes until flower budding were smaller in FR than in B, while flower budding was 
not observed in SD during the same period (Table 1). The results reveal that both monochromatic far-red and blue light promote flowering, with far-red being the more effective.

Flowering of E. grandiflorum has been shown to be enhanced under low red:far-red ratios (Sato et al., 2009; Yamada et al., 2008; 2009). Furthermore, the flowering of G. paniculata was promoted to a greater degree by far-red light supplemented with weak red light compared to monochromatic far-red light (Nishidate et al., 2012). Therefore, a comparison between the promotion of flowering in E. grandiflorum by monochromatic far-red light and that by far-red light supplemented with red light would be interesting. The number of days to flower budding was not reportedly different between the night-break treatment with $\mathrm{B}$ and the non-treatment control in E. grandiflorum, whereas the fewer nodes arising in the treatment with B suggests the possibility of the promotion of flowering by B (Yamada et al., 2011). The promotion of blue light became clear in this study, although its effect appears to be weaker than that of far-red light.

\subsection{Expression analysis of flowering-related genes}

We compared changes in flower budding and the expression of the flowering-related genes EgFTL and EgSOC1L with FR and B on ZT8, which represents the end of photoperiod under sunlight, according to Hori et al. (2011) (Fig. 1). During the period of LED radiation, the levels of EgFTL and EgSOC1L in SD were lower than those in FR and B. The level of $E g F T L$ mRNA increased 4 weeks after the start of LED radiation, reaching a peak after 8 weeks in FR and 10 weeks in $\mathrm{B}$. The level of EgSOC1L mRNA was higher in FR than in B and SD after 4 weeks (peaking at 8 weeks), and higher in B than in SD after 10 weeks. The onset of floral primordia is about 20 days before flower budding under long-day conditions (Islam et al., 2005; Tsukada, 2003); therefore, we focused on the gene expression preceding the expected date of flower bud differentiation. Flower bud differentiation was expected to occur at 8.7 and 11.7 weeks after the start of treatment in FR and B (Table 1), respectively, and both $E g F T L$ and $E g S O C 1 L$ showed peaks preceding those.

The expression of the E. grandiflorum FLC homolog EgFLCL was also analyzed as another potential flowering-related gene because $E g F L C L$ shows a unique flowering response to temperature, which is different from what happens in Arabidopsis (Nakano et al., 2011), and the expression of FLC is affected by light quality (Golembeskia and Imaizumi, 2015). We found that neither FR nor B affected the level of EgFLCL

Table 1. Effect of light quality on days to flower budding and node numbers of first flower bud in E. grandiflorum.

\begin{tabular}{lll}
\hline $\begin{array}{l}\text { Light } \\
\text { source }\end{array}$ & $\begin{array}{l}\text { Days to flower } \\
\text { budding }\end{array}$ & $\begin{array}{l}\text { Node number of } \\
\text { first flower buds }\end{array}$ \\
\hline FR & 81.0 & 11.5 \\
B & 102.0 & 15.6 \\
$t$-test & $* *$ & $* *$ \\
\hline
\end{tabular}

Days to flower budding after the start of LED radiation were shown. Values are expressed as mean $(\mathrm{n}=8)$ and $* *$ indicates a significant difference at $P<0.01$ according to the $t$-test.
mRNA (Fig. 1). Nakano et al. (2011) reported that the expression of $E g F L C L$ is not affected by photoperiod and our work using monochromatic light confirmed the previous study's result.

The effect of LED radiation treatment under dark conditions on the expression of FT and SOC1 or their homologs has been previously reported in Arabidopsis and G. paniculata (Hori et al., 2011; Shibuya et al., 2017). According to these studies, SOC1 expression was induced by far-red light, FT and SOC1 expressions were induced by blue light, and flowering was
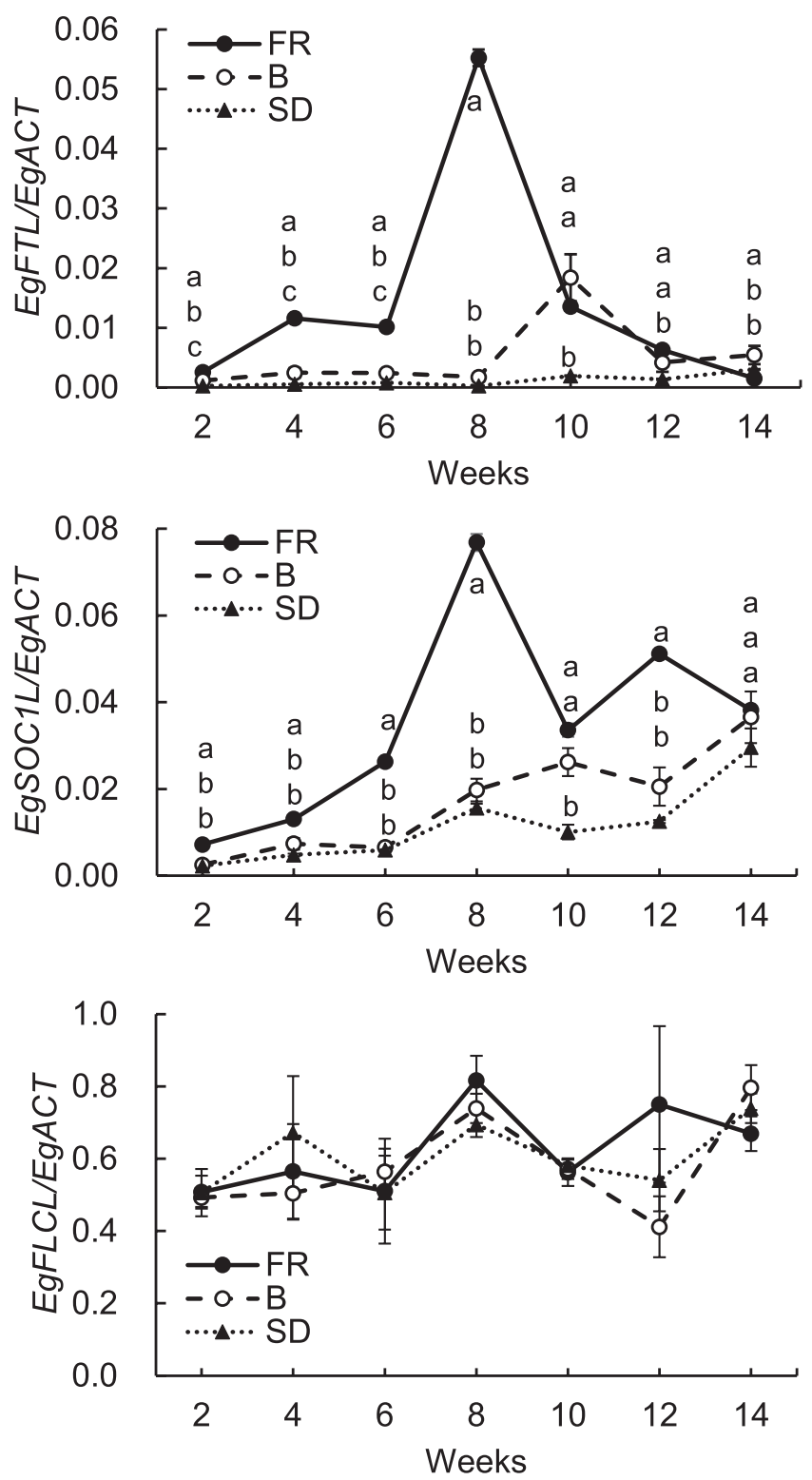

Fig. 1. Changes in expression of the flowering-related genes, $E g F T L, E g S O C 1 L$, and $E g F L C L$, after LED radiation treatment was started in E. grandiflorum. The relative expression levels were calculated on the basis of real-time PCR data. Values are expressed as mean \pm SE of three biological replicates $(n=$ 3). Values with different letters in each week are significantly different at $P<0.05$ according to the Tukey-Kramer test. Values in $E g F L C L$ are not significantly different in all weeks. 
promoted by both far-red and blue light in Arabidopsis. In contrast, the expression of FT and SOC1 homologs was induced by both far-red light and blue light in G. paniculata 'Million Star', which showed the same flowering response to light quality. In addition, although flowering was promoted by both far-red and blue light in long-day strawberry plants, the expression of $F T$ homolog was induced only by blue light (Yoshida et al., 2016). In the present study, the levels of EgFTL and EgSOC1L showed peaks preceding flower bud differentiation in FR and $\mathrm{B}$, suggesting that both genes play a role in flowering in $E$. grandiflorum, an effect similar to that seen in G. paniculata 'Million Star'. In addition, the later expression of these genes by B in E. grandiflorum compared to that by FR could be associated with weak promotion of flowering by blue light. There is a possibility that EgFTL expression and flowering induction are caused by $\mathrm{CO}$ stabilization via phyA or crys functions in SPA1-COP1 complex formation.

\subsection{Expression analysis of light quality-responsive genes}

Although there is much less information at the molecular level for E. grandiflorum compared to Arabidopsis, a few light quality-responsive genes have been reported in relation to the early development of E. grandiflorum seedlings (Takemura et al., 2015). Among those genes, we analyzed the expression of genes related to gibberellin (GA) and regulation of transcription, for which roles in photoperiodic flowering are unknown (Fig. 2). Although the mRNA levels of the putative GA metabolism-related genes EgGA20ox and EgGA2ox were affected by light quality at some stages, they did not appear to correspond to those of EgFTL and EgSOC1L. As Nakano et al. (2011) have suggested that the GA pathway is possibly related to the regulation of flowering by vernalization and photoperiod, expression of GA metabolism-related genes was analyzed in this study and the previous study using the EgFTL overexpressor ( $\mathrm{Li}$ et al., 2015). However, the coordination of expression of GA metabolism-related genes and EgFTL was not observed in either study. On the other hand, the mRNA level of $E g B T B / P O Z 1$ showed a peak with FR after 8 weeks with $E g F T L$. Although there are some reports on the role of $\mathrm{BTB} / \mathrm{POZ}$ domain-containing protein in flowering (Chen et al., 2015; Xu et al., 2016), information is lacking on the role of $\mathrm{BTB} / \mathrm{POZ}$ domain-containing protein in the photoperiodic regulation of flowering and its effect on the ratio of phyB-Pfr/Pr in the FR-mediated promotion of flowering. Therefore, further studies are required.

The expression of the light quality-responsive genes and $E g F L C$ was evaluated simultaneously with those in EgFTL, assuming the regulation of EgFTL expression by their genes. It has been reported that the expression of $F L C$ and GA-related genes is relatively high at similar periods under long-day conditions (Hisamatsu et al., 2005; Yasui et al., 2012), although their long-day treatment is not necessarily similar to that in the present study. The expression of FLC and GA-related genes did not correspond to $F T$ expression and flowering in the present study. Nevertheless, their involvement may not be ruled out due to lack of information on their expression rhythms.
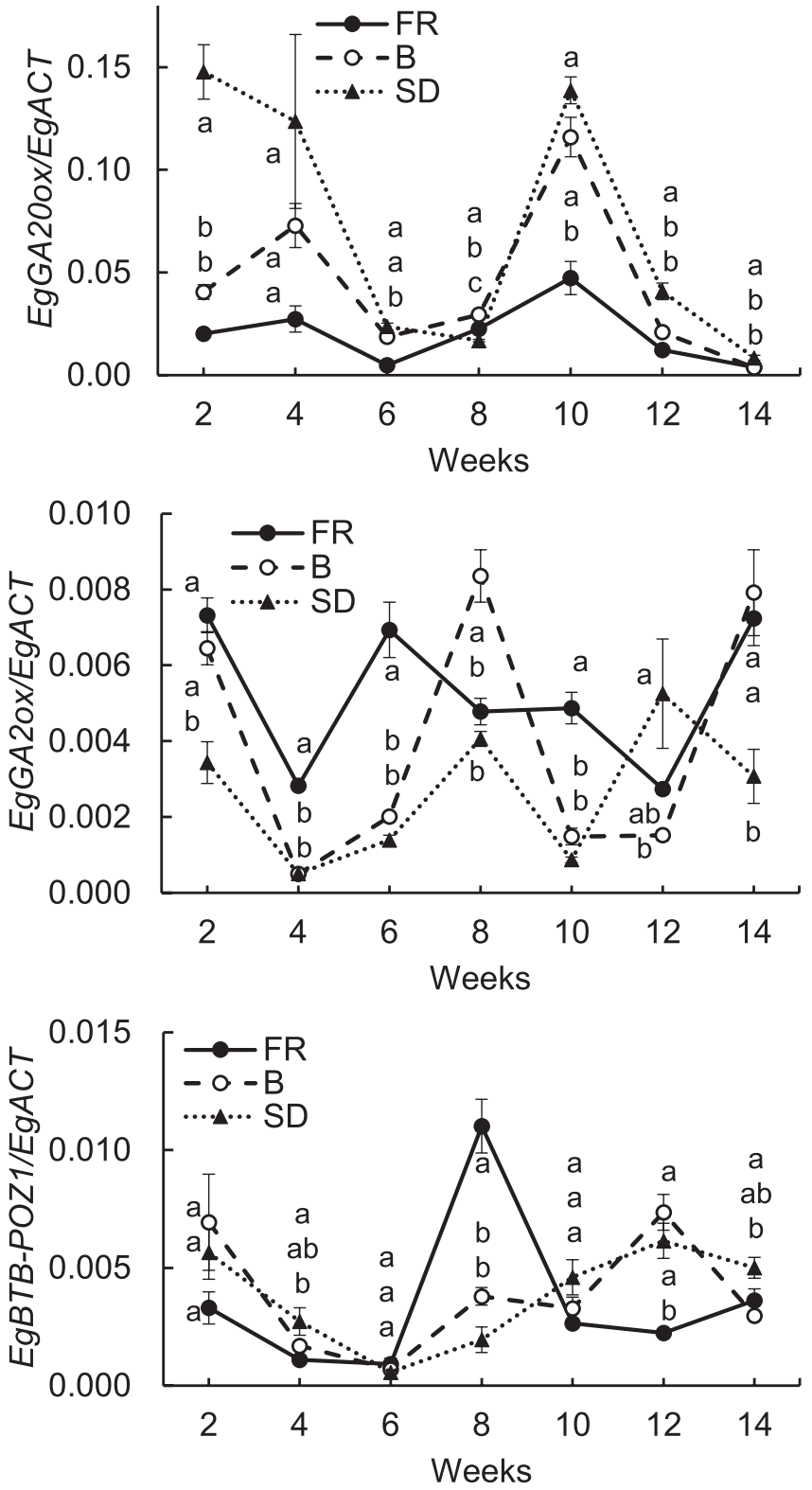

Fig. 2. Changes in expression of the light quality-responsive genes, EgGA20ox, EgGA2ox, and EgBTB-POZ1, after LED radiation treatment was started in $E$. grandiflorum. The relative expression levels are calculated on the basis of real-time PCR data. Values are expressed as mean \pm SE of three biological replicates $(n=3)$. Values with different letters in each week are significantly different at $P<0.05$ according to the Tukey-Kramer test.

\subsection{Concluding remarks}

The effect of monochromatic light on flower budding was investigated in E. grandiflorum using FR and B, while flowering is inhibited by red LEDs (Yamada et al., 2011). Flower budding was promoted by both, though somewhat more so with far-red than blue light. The flowering promotion effect of blue light and far red light as monochromatic light was clearly shown with evidential gene expression data for the first time here. FR reduced stem diameter and SPAD values in E. grandiflorum (data not shown); therefore, the use of blue light is an option if 
cut flower quality is a problem under far-red light. In an earlier assessment, expression of the SOC1 homolog was found to be induced by far-red, and expression of neither the SOC1 homolog nor the FT homolog was induced by blue light in G. paniculata 'Bristol Fairy', in which flowering was promoted by far-red light but not blue light (Hori et al., 2011). A possible mechanism for this flowering response to blue light may be associated with variation in nucleotide sequences of the promoter region of the $F T$ homolog, rather than with the function of the homologs of FKF1 and GI, which are involved in blue light signaling (Shibuya et al., 2017). Conversely, in the present study, changes in the levels of both EgFTL and EgSOC1L corresponded to the flowering response in $\mathrm{FR}$ and $\mathrm{B}$, suggesting that a variable response of a common gene upstream of EgFTL and EgSOC1L to far-red and blue lights result in differences in flowering response to these wavelengths. In situ analysis of mRNA localization of these genes will be necessary to confirm their function.

\section{Acknowledgment}

This work was supported by Grants-in-Aid for Scientific Research [24248006].

\section{References}

Casal JJ, Sanchez RA, Yanovsky MJ, 1997: The function of phytochrome A. Plant, Cell and Environment 20, 813-819.

Chen L, Bernhardt A, Lee JH, Hellmann H, 2015: Identification of Arabidopsis MYB56 as a novel substrate for CRL3 ${ }^{\mathrm{BPM}}$ E3 ligases. Molecular Plant 8, 242-250.

Eskins K, 1992: Light quality effects on Arabidopsis development. Red, blue and far-red regulation of flowering and morphology. Physiologia Plantarum 86, 436-444.

Golembeskia GS, Imaizumi T, 2015: Photoperiodic regulation of florigen function in Arabidopsis thaliana. Arabidopsis Book 13, e0178.

Hamamoto H, Shimaji H, Higashide T, 2003: Budding and bolting responses of horticultural plants to night-break treatments with LEDs of various colors. Journal of Agricultural Meteorology 59, 103-110.

Hikosaka S, Iyoki S, Hayakumo M, Goto E, 2013: Effects of light intensity and amount of supplemental LED lighting on photosynthesis and fruit growth of tomato plants under artificial conditions. Journal of Agricultural Meteorology 69, 93-100.

Hisamatsu T, King RW, Helliwell CA, Koshioka M, 2005: The involvement of gibberellin 20-oxidase genes in phytochrome-regulated petiole elongation of Arabidopsis. Plant Physiology 138, 1106-1116.

Hori Y, Nishidate K, Nishiyama M, Kanahama K, Kanayama Y, 2011: Flowering and expression of flowering-related genes under long-day conditions with light-emitting diodes. Planta 234, 321-330.

Islam N, Patil G, Gislerød H, 2005: Effect of photoperiod and light integral on flowering and growth of Eustoma grandiflorum (Raf.) Shinn. Scientia Horticulturae 103, 441-451.

Jung J, Ju Y, Seo P, Lee J, Park C, 2012: The SOC1-SPL module integrates photoperiod and gibberellic acid signals to control flowering time in Arabidopsis. The Plant Journal 69, 577-588.
Khan N, Abas N, 2011: Comparative study of energy saving light sources. Renewable and Sustainable Energy Reviews 15, 296-309.

Li HL, He SB, Zhang YC, Zhu DM, Zhang JY, 2011: Blue-light-dependent interaction of cryptochrome 1 with SPA1 defines a dynamic signaling mechanism. Genes \& Development 25, 1023-1028.

Li KH, Chuang TH, Hou CJ, Yang CH, 2015: Functional analysis of the FT homolog from Eustoma grandiflorum reveals its role in regulating $\mathrm{A}$ and $\mathrm{C}$ functional MADS box genes to control floral transition and flower formation. Plant Molecular Biological Reporter 33, 770-782.

Liu B, Zuo Z, Liu H, Liu X, Lin C, 2011: Arabidopsis cryptochrome 1 interacts with SPA1 to suppress COP1 activity in response to blue light. Genes \& Development $\mathbf{2 5}$, 1029-1034.

Miyamae H, Shima K, Nishitani T, Yamada M, Ishiwata M, Sumitomo K, Hisamatsu T, 2015: Effect of Spectral distributions of the red-to-far-red wavelength range on flowering and extension growth of stock (Matthiola incana (L.) R. Br.). Horticultural Research (Japan) 14, 371-379 (in Japanese with English abstract).

Murakami K, Matsuda R, Fujiwara, K, 2013: Effects of supplemental lighting to a lower leaf using light-emitting diodes with different spectra on the leaf photosynthetic rate in sweet pepper. Journal of Agricultural Meteorology 69, 55-63.

Nakano Y, Kawashima H, Kinoshita T, Yoshikawa H, Hisamatsu T, 2011: Characterization of FLC, SOC1 and FT homologs in Eustoma grandiflorum: effects of vernalization and post-vernalization conditions on flowering and gene expression. Physiologia Plantarum 141, 383-393.

Nishidate K, Kanayama Y, Nishiyama M, Yamamoto T, Hamaguchi Y, Kanahama K, 2012: Far-red light supplemented with weak red light promotes flowering of Gypsophila paniculata. Journal of the Japanese Society for Horticultural Science 81, 198-203.

Sato T, Kudo N, Moriyama T, Ohkawa H, Kanayama Y, Kanahama K, 2009: Acceleration of flowering of Eustoma grandiflorum in early winter by day-extension treatments with far-red rich bulb-type fluorescent lamps. Horticultural Research (Japan) 8, 327-334 (in Japanese with English abstract).

Sheerin D, Menon C, zur Oven-Krockhaus S, Enderle B, Stierhof YD, Huq E, Hiltbrunner A, 2015: Light-activated phytochrome A and B interact with members of the SPA family to promote photomorphogenesis in Arabidopsis by reorganizing the COP1/SPA complex. The Plant Cell 27, 189-201.

Shibuya T, Kanayama Y, 2014: Flowering response to blue light and its molecular mechanisms in Arabidopsis and horticultural plants. Advances in Horticultural Science 28, 179-183.

Shibuya T, Murakawa Y, Nishidate K, Nishiyama M, Kanayama Y, 2017: Characterization of flowering-related genes and flowering response in relation to blue light in Gypsophila paniculata. The Horticulture Journal 86, 94-104.

Srikanth A, Schmid M, 2011: Regulation of flowering time: all roads lead to Rome. Cell Molecular Life Science 68, 2013-2037.

Takemura Y, Kuroki K, Katou M, Kishimoto M, Tsuji W, Nishihara E, Tamura F, 2015: Gene expression changes triggered by end-of-day far-red light treatment on early developmental stages of Eustoma grandiflorum (Raf.) Shinn. 
Scientific Reports 5, 17864.

Tsukada A, 2003: Seiikutoseiriseitai Natsugata, Yusutoma. In Nougyougijyutsutaikei Kakihen 8 (ed. by Nousangyosonbunkakyoukai). Nousangyosonbunkakyoukai, Tokyo, pp. 391-396 (in Japanese).

Xu C, Park SJ, Eck JV, Lippman ZB, 2016: Control of inflorescence architecture in tomato by $\mathrm{BTB} / \mathrm{POZ}$ transcriptional regulators. Genes \& Development $\mathbf{3 0}$, 2048-2061.

Yamada A, Tanigawa T, Suyama T, Mastuno T, Kunitake T, 2008: Improvement of Eustoma grandiflorum (Raf.) Shin. cut flower quality for early-autumn shipping with long-day treatment using light sources that delay flower bud formation. Journal of the Japanese Society for Horticultural Science 77, 296-303.

Yamada A, Tanigawa T, Suyama T, Matsuno T, Kunitake T, 2009: Red: far-red light ratio and far-red light integral promote or retard growth and flowering in Eustoma grandiflorum (Raf.)
Shin. Scientia Horticulturae 120, 101-106.

Yamada A, Tanigawa T, Suyama T, Matsuno T, Kunitake T, 2011: Effects of red:far-red light ratio of night-break treatments on growth and flowering of Eustoma grandiflorum (Raf.) Shinn. Acta Horticulturae 907, 313-317.

Yasui Y, Mukougawa K, Uemoto M, Yokofuji A, Suzuri R, Nishitani A, Kohchi T, 2012: The phytochrome-interacting VASCULAR PLANT ONE-ZINC FINGER1 and VOZ2 redundantly regulate flowering in Arabidopsis. The Plant Cell 24, 3248-3263.

Yoshida H, Mizuta D, Fukuda N, Hikosaka S, Goto E, 2016: Effects of varying light quality from single-peak blue and red light-emitting diodes during nursery period on flowering, photosynthesis, growth, and fruit yield of everbearing strawberry. Plant Biotechnology 33, 267-276.

Zuo Z, Liu H, Liu B, Liu X, Lin C, 2011: Blue light-dependent interaction of CRY2 with SPA1 regulates COP1 activity and floral initiation in Arabidopsis. Current Biology 21, 841-847. 